Review Article

\title{
Actin-Tethered Junctional Complexes in Angiogenesis and Lymphangiogenesis in Association with Vascular Endothelial Growth Factor
}

\author{
Dimitar P. Zankov and Hisakazu Ogita \\ Division of Molecular Medical Biochemistry, Department of Biochemistry and Molecular Biology, \\ Shiga University of Medical Science, Seta Tsukinowa-cho, Shiga, Otsu 520-2192, Japan \\ Correspondence should be addressed to Dimitar P. Zankov; dzankoff@belle.shiga-med.ac.jp \\ and Hisakazu Ogita; hogita@belle.shiga-med.ac.jp
}

Received 11 September 2014; Revised 23 October 2014; Accepted 31 October 2014

Academic Editor: Qiang Zhao

Copyright ( 2015 D. P. Zankov and H. Ogita. This is an open access article distributed under the Creative Commons Attribution License, which permits unrestricted use, distribution, and reproduction in any medium, provided the original work is properly cited.

\begin{abstract}
Vasculature is present in all tissues and therefore is indispensable for development, biology, and pathology of multicellular organisms. Endothelial cells guarantee proper function of the vessels and are the original component in angiogenesis. Morphogenesis of the vascular system utilizes processes like cell adhesion, motility, proliferation, and survival that are closely related to the dynamics of actin filaments and actin-tethered adhesion complexes. Here we review involvement of actin cytoskeletonassociated junctional molecules of endothelial cells in angiogenesis and lymphangiogenesis. Particularly, we focus on F-actin binding protein afadin, an adaptor protein involved in broad range of signaling mechanisms. Afadin mediates the pathways of vascular endothelial growth factor- (VEGF-) and sphingosine 1-phosphate-triggered angiogenesis and is essential for embryonic development of lymph vessels in mice. We propose that targeting actin-tethered junctional molecules, including afadin, may present a new approach to angiogenic therapy that in combination with today used medications like VEGF inhibitors will benefit against development of pathological angiogenesis.
\end{abstract}

\section{Introduction}

Endothelial cells (ECs) in mature vascular system are quiescent, nonproliferating (with some exceptions, e.g., uterus) heterogenic population. The endothelium generated by a single layer of ECs separates the blood and lymph from other components of the vessel wall and serves wide variety of functions, specific not only for the vascular bed but also for the tissue they populate $[1,2]$. ECs are the first component of blood vasculature that is formed in the embryo by differentiation of mesodermal precursor cells angioblasts (process defined as vasculogenesis, VG), thus creating the primary capillary plexus [3]. Subsequently, the embryonic vasculature evolves from the existing vessels by remodeling (termed as angiogenesis, AG) [4]. In contrast, lymphangiogenesis (LAG) starts with migration, proliferation, and differentiation of ECs pool residing in cardinal vein $[5,6]$.

Intercellular junctions between the adjacent ECs and between ECs and surrounding non-EC wall components (e.g., pericytes) maintain the organization of EC layer and vessel integrity. Their function is beyond just mechanical support involving at least inhibition of ECs proliferation and neovasculogenesis in mature vessels as well as regulation of ECs gene expression and survival [7]. Adhesive machinery of ECs includes adherens and tight junctions and focal adhesions [8], all associated with intracellular F-actin network. Morphogenesis of vasculature relies on processes like cell adhesion, motility, and proliferation that inevitably include the actin cytoskeleton and associated junctional molecules, making the majority of these complexes a requisite of $\mathrm{VG}$, AG, and LAG [9-14].

In this review we focus on the involvement of actinassociated molecules at the junctional apparatus in AG and LAG and, in particular, afadin, an adaptor protein with multiple roles in cellular physiology [15]. Small GTP-binding proteins (GTPases) Rapl and RhoA are discussed in the context of afadin signaling. The role of GTPases related to actin cytoskeleton organization and AG is beyond the 
scope of this paper. The interested readers may refer to a number of outstanding publications [16-18]. We have selected this particular view on vascular development, because those adherent complexes are deeply interwoven with the signaling of the "prime switches" of AG: vascular endothelial growth factors (VEGFs) and their receptor-tyrosine kinase VEGF receptors [19], which makes them appealing target for pro/antiangiogenic therapy.

\section{Afadin in the Pathways Controlling AG and LAG}

Afadin is an adaptor protein discovered in 1997 by Mandai et al. and holds two RA (Ras association), a FHA (forkheadassociated), a DIL (dilute), a PDZ (postsynaptic density, Drosophila disk large tumor suppressor, zonula occludens1), three PR (proline-rich), and F-actin structural domains (Figure 1) [20]. Two isoforms are described at present: 1afadin and s-afadin. s-Afadin truncates the C-terminal Factin and the third PR domains. l-Afadin is expressed ubiquitously, whereas s-afadin is expressed mainly in the nerve tissue [21]. F-actin and PDZ domains link actin filaments and Ig-like transmembrane junctional proteins nectins, respectively. Resulting cell-cell adhesion assembly is crucial for establishment and part of adherens and tight junctions in epithelia, fibroblasts, and ECs $[15,22]$. In addition, afadin functions independently of nectins to promote cell movement and neuronal physiology [23-26]. Due to the multitude of interacting domains and fundamental role of cell-cell junctions for tissue organization [27], afadin is involved in various biological phenomena ranging from embryonic development to cancer progression. Complexity of those processes creates a broad field of constantly increasing information of afadin roles [28-32].

Physiological AG, the formation of blood vessels from existing ones, occurs not only in the embryo but also in postnatal life (e.g., in uterus, during wound healing). Pathological AG accompanies some chronic inflammatory diseases (e.g., rheumatoid arthritis), cancer, and atherosclerosis [4, 33, 34]. During physiological AG, there is fine-tuned balance between stimulating and suppressing factors in order to maintain vascular and tissue integrity and assure effective vessel formation [35]. Pathological AG results in disorganized, abnormal vasculature with disturbed regulation [4].

Undeniably, the prime molecular machinery that stimulates VG and sprouting AG is comprised of VEGF and VEGF receptor in ECs $[4,33,34,36]$. VEGF receptor interacts with key ECs adhesion molecules (e.g., VE-cadherin, neuropilin, and integrins), guides tip ECs to VEGF signal, and activates a myriad of intracellular signaling during all phases of AG. Genetic inhibition of VEGF or VEGF receptor in mice prevents successful vessel formation and cause embryonic death [37-39].

VEGF signaling is also critical for tumor AG. At present the most extensively applied medication in human cancer treatment is VEGF inhibitors [40]. One of the downstream targets of activated VEGF receptor is Rapl GTPase that is also indispensable for the vessel formation [41]. In epithelial cells Rap1 associates with afadin and recruits epithelial (E)cadherin to adherens junctions [42]. Understanding of the partners of activated Rapl in ECs had not been extensive when we investigated Rapl-driven mechanisms in VEGF and sphingosine 1-phosphate- (S1P-) induced AG [43]. By studying VEGF- or S1P-stimulated human umbilical vein ECs (HUVECs) and conditional knockout (cKO) mice with endothelial-specific afadin gene disruption, we found that (i) in HUVECs, intracellular localization of afadin was Rap1dependent and colocalization of activated (GTP-containing) Rapl and afadin was observed in the cell-cell contacts and the leading edge of polarized moving cells; (ii) afadin or Rap1 knockdown in HUVECs reduced VEGF- or S1P-stimulated capillary-like network formation in Matrigel and 3D gels, suppressed migration and proliferation of HUVECs, and increased the number of apoptotic cells; (iii) equivalent to the epithelial cells, Rapl and afadin played key roles in accumulation of adherens and tight junction proteins since absence of afadin or Rapl in HUVECs removed the fluorescent signal in the cell membrane for nectin-2, VE-cadherin, claudin5, and junctional adhesion molecule A; (iv) in VEGF- or S1P-stimulated HUVECs, afadin and Rap1 controlled specifically phosphorylation of Akt and endothelial nitric oxide synthase (eNOS) but not extracellular signal-regulated kinase or $\mathrm{p} 38$. The subsequent experiments revealed that afadin is essential for the interaction between phosphoinositide 3kinase (PI3K) regulatory subunit p85 and VEGF or S1P receptors. That interaction recruits catalytic subunit p110 of PI3K and Akt/eNOS phosphorylation follows (schematically drawn in Figure 2). Akt/eNOS signaling is proangiogenic and downstream of VEGF and S1P in ECs [44, 45].

We have also found that, in endothelial afadin cKO mice, postnatal development of retinal vessels is initially delayed, that small vessels network decreased, and that VEcadherin staining in ECs became discontinuous. Even in heterozygous $\mathrm{KO}$ mice, recovery of blood flow and neoformation of capillary networks after hind limb ischemia were diminished compared to control mice. Those in vivo data validated the vital role of afadin in postnatal AG. Moreover, afadin is involved in maintaining epithelial and endothelial barrier function [46-48], migration of cancer cells [29, 32], elongation and lumen formation of the developing nephron [49], and modulation of integrin levels in epithelial cells [50]. All those findings provide evidence that afadin is one of the main participants in the mechanisms of cellular adhesion, motility, and proliferation, processes implicated in AG but also in a broad variety of other physiological and pathological events.

VEGF receptor-Rap1 activation signal in ECs is transmitted not only to afadin/Akt but also to p42/44 ERK1/2 and p38 MAPK [51]. Those tyrosine kinases are important for ECs proliferation and actin cytoskeleton remodeling and are absolutely required for placental AG $[52,53]$. In Raplbdeficient mice AG is disturbed resulting in embryonic and perinatal mortality [54]. The mechanisms for the defective AG comprise ECs dysfunction (decreased activation, migration, and proliferation) that coincides with lower levels of p42/44 ERK1/2 and p38 MAPK in ECs. Another downstream target of Rapl in ECs is RAPL, protein that associates with 


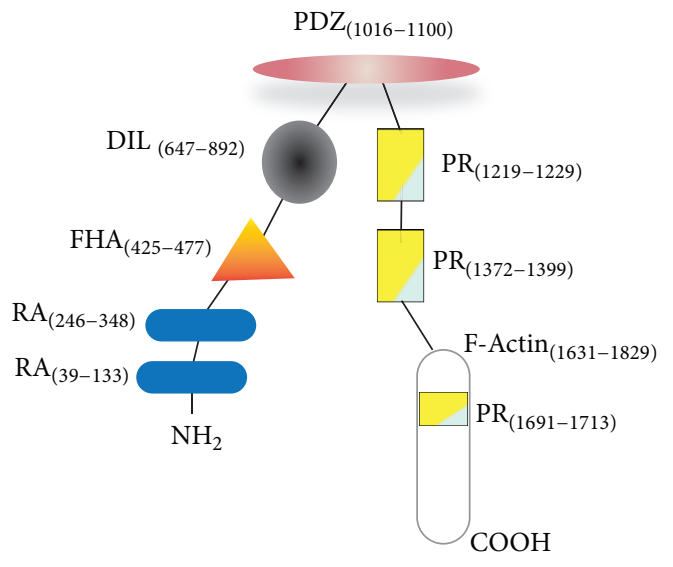

1-Afadin

(a)

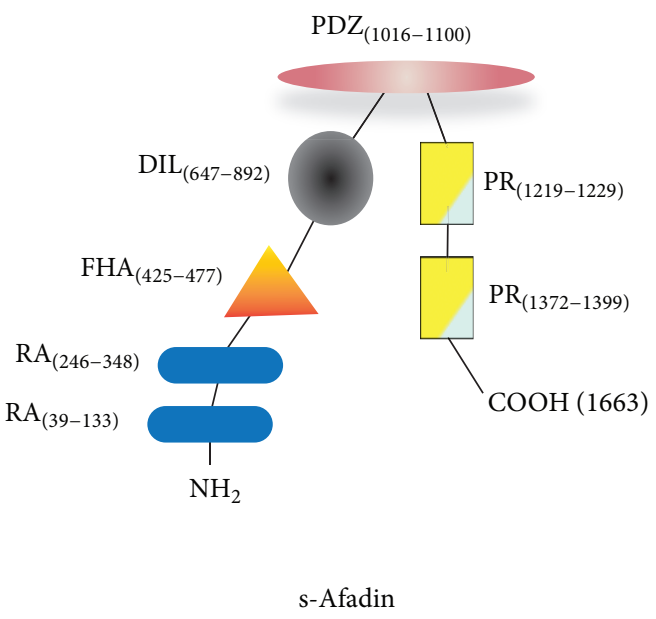

(b)

FIGURE 1: Anatomy of afadin molecule. The modular structure of l-afadin (a) and s-afadin (b) is schematically shown. Numbers in parentheses indicate the first and last amino acid of the structural domains. RA: Ras associated domain; FHA: forkhead associated domain; DIL: dilute domain; PDZ: postsynaptic density, Drosophila disk large tumor suppressor, zonula occludens-1 domain; PR: proline rich domain; F-actin: F-actin binding domain. PDZ domain interacts with nectin molecules.

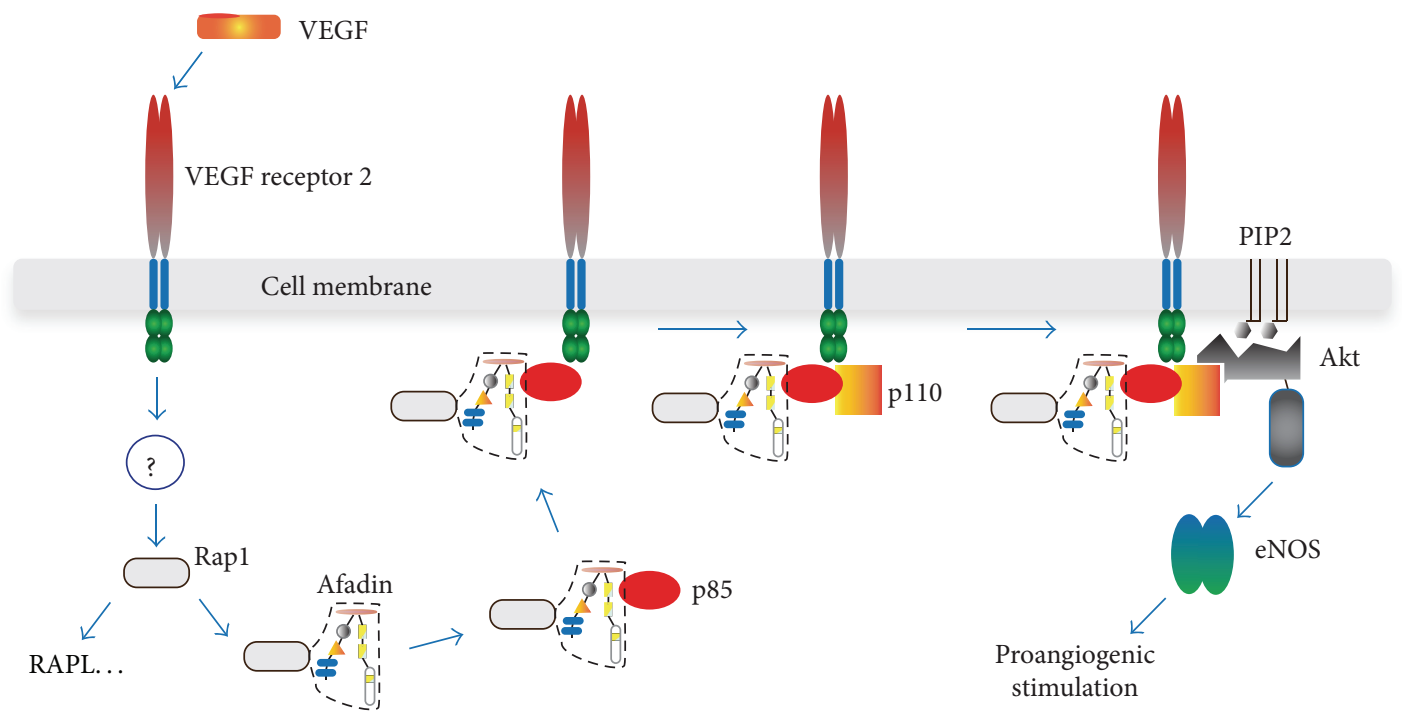

FIgURE 2: Proposed function of afadin in the VEGF or S1P receptor signaling during AG. In HUVECs, activated Rap1 (by still unknown mechanism) binds and recruits afadin to cell membrane where the complex between VEGF receptor, afadin, Rap1, and sequentially p85 and p110 subunits of PI3K assembles. Activated PI3K phosphorylates Akt and downstream signaling follows. Similar events occur after S1P receptor activation (not drawn). In addition, Rapl stimulates different proteins (e.g., RAPL) that may contribute to proangiogenic signal.

GTP-bound Rap1 to activate integrins [55]. This pathway has proved essential in angiogenic sprouting, ECs migration (HUVECs), adhesion, and in vivo neovascularization (hind limb ischemia model in mice) [56]. The role of RAPL in AG is attributed to inside-out integrin signaling: RAPL promotes $\beta 1$ integrin affinity, thereby stimulating ECs adhesion and migration (for the role of integrins in AG and LAG, see the next section of this review).

The mice with EC-targeted deletion of afadin [43] were born in a significantly reduced ratio $(3.6 \%$ versus $25 \%$ expected), showing that afadin is also important for embryonic AG. That observation became the topic of our investigation [57], in which we found that most of the endothelial afadin cKO mouse embryos died at embryonic day (E) 16.5. Until E13.5, no detectable dissimilarities between cKO and control embryos could be observed, but at E14.5-E16.5, cKO mice developed diffuse subcutaneous edema and dot-like skin hemorrhages. Series of immunofluorescence experiments demonstrated that lymphatic vessels in the skin of afadin cKO embryos were largely dilated and that lymphatic endothelium exhibited defect in VEcadherin staining but preserved ability to differentiate and 


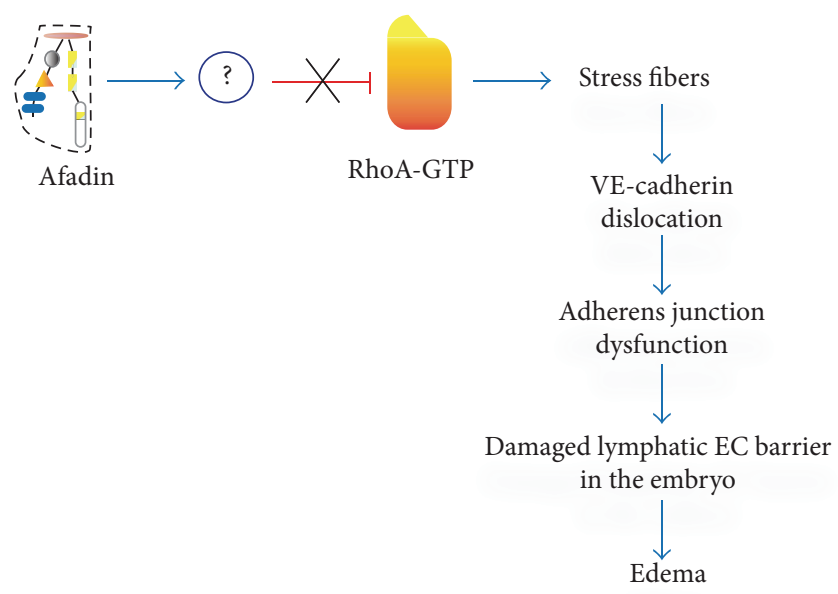

FIGURE 3: Sequence of regulatory steps leading to damage of lymph endothelial barrier in afadin cKO mouse embryos. In the absence of afadin-mediated inhibition of RhoA activity, actin stress fibers are formed. Thick actin filaments alter the cell shape, dislocate VE-cadherin from cell membrane, compromise adherent junctions, and damage lymph EC barrier. This results in generalized edema and embryonic death.

proliferate. To investigate afadin-induced dysfunction of lymphatic endothelium, human dermal blood and lymphatic microvascular ECs (BMVECs and LMVECs, resp.) were used as a model. Knockdown of afadin in LMVECs triggered cell-shape alteration, disorganization of cell-cell contacts, reduction of VE-cadherin staining, and formation of thick F-actin fibers at the cells periphery. All of these effects were not produced in BMVECs. Afadin-associated F-actin/VEcadherin rearrangements in LMVECs depended on RhoA activity: GTP-bound (active) RhoA was increased in afadinknockdown LMVECs and dominant negative RhoA mutant rescued the phenotype in LVMECs. Thus, afadin stabilizes adherens junctions/VE-cadherin by suppressing RhoA activity and stress fibers formation to maintain EC barrier in embryonic lymphatic vessels (Figure 3). Actin fibers organization is reported as condition that defines the localization of VE-cadherin in cell junctions. Actin depolymerization or hyperpolymerization has been shown to decrease VEcadherin in ECs and compromise endothelial barrier [58]. Contraction of cortical actin-myosin cytoskeleton in ECs stimulated by Rho GTPase has similar effect [59]. Actin bundles in ECs formed after cAMP/Epac/Rap1 stimulation anchor VE-cadherin and strengthen cell-cell adhesions, the effect that results in reduction of endothelial layer permeability $[60,61]$.

Our reports described above complement the knowledge about signaling in embryonic LAG and postnatal AG. Lack of afadin in lymphatic ECs of the mouse embryo does not impede vessel morphogenesis but compromises intercellular junctions of ECs. This effect is secondary to F-actin stress fiber synthesis and VE-cadherin dislocation. Contrary to adhesion structures in blood ECs, lymphatic ECs have "button-like" intercellular contacts that facilitate the transport of intercellular fluids [62]. This functional specialization may also include unique RhoA regulation by afadin. In addition, RhoA-dependent actin rearrangement and disruption of cell-cell adherens junction resemble the mechanism exploited by thrombin to damage barrier function in lung ECs [47]. Afadin/Rap1 complex was essential for recovery of cell-cell contacts and actin network in lung ECs.

Participation of afadin in the signaling downstream of VEGF and S1P receptors as well as its involvement in tubulogenesis and apoptosis might focus attention on this molecule in the context of tumor AG and cancer therapy. Afadin is also important antiapoptotic guard in embryogenesis, and platelet-derived growth factor (PDGF) receptor binds PI3K regulatory subunit $\mathrm{p} 85$ /afadin in a similar way as shown for VEGF and S1P receptors to activate Akt [63].

\section{Other Actin-Associated Adhesion Complexes in relation to AG and LAG}

Many transmembrane adhesion molecules in ECs and their intracellular partners that link the molecular assembly to actin filaments contribute to the complex sequence of events in the process of VEGF-induced AG. Studies with cKO mice that eliminate the function of selected molecule in ECs demonstrated the individual role of excluded junctional component in AG or LAG and revealed its unique phenotype.

3.1. VE-Cadherin. VE-cadherin communicates through its cytoplasmic segment with $\beta$-catenin, plakoglobin, and p120catenin. $\beta$-Catenin and plakoglobin interact with $\alpha$-catenin that anchors (although recently questioned [64]) VEcadherin to actin cytoskeleton $[65,66]$. p120-Catenin stabilizes VE-cadherin by preventing its endocytosis [67]. VEcadherin deficiency in ECs of mice created by cKO technique has caused embryonic lethality at E9.5 because of increased EC apoptosis and defective AG [68]. VG was not disturbed, but lack of VE-cadherin stopped the remodeling of primary capillary plexus. Some vessels of the embryos had narrow or 
no lumen, whereas others were dilated. Throughout the vasculature, ECs were detached from the basement membranes, disconnected each other, and could be seen in the lumen of the vessels. Those observations resulted from a failure to form the EC survival complex amid VEGF, VE-cadherin, $\beta$ catenin, and $\mathrm{PI} 3 \mathrm{~K}$, missing Akt phosphorylation and thus inactive antiapoptotic machinery [69].

VE-cadherin is crucial for the functional integrity of endothelial layer [70] and contributes to all phases of AG. Upon initiation of VEGF-mediated AG and activation of VEGF receptor, VE-cadherin is target for Src phosphorylation, a modulation that leads to adherens junction disassembly and increased permeability of ECs. Simultaneously, molecular complex consisting of integrin $\alpha \mathrm{v} \beta 3$, syndecan -1 , and insulin-like growth factor-1 receptor is activated. These molecular events facilitate early adhesion and migration of ECs in VEGF-induced AG [71]. During sprouting neovascularization, the tip and stalk ECs are recognized in the developing branches [72]. It has been found that tip and stalk ECs are not static but they exchange their positions and phenotypes in the process of elongation of the vessel [73]. VE-cadherin dynamics under contrasting control of VEGF and Notch signaling drives this ECs behavior and is necessary for the coordination of physiological AG. VEcadherin mobility is lost in certain pathology, for example, cancer [74]. VE-cadherin is important for establishing the functional vessels by inhibiting VEGF-signaling (p44/p42 MAPK) and ECs proliferation after ECs contact and adherens junction formation [75].

3.2. $\beta$-Catenin. Mice embryos with inactivated $\beta$-catenin in ECs die at E11.5-E13.5 [10]. VG and early AG developed, but after E9.5 vessels showed lumen irregularities and hemorrhage. Vitelline vessels had smaller diameter. The pattern of the vasculature of primitive neural plexus was defective, and the placenta was less vascularized compared to control mice. ECs had morphological changes, impaired junctions, and abundant fenestrations. In cultured ECs of $\beta$-catenin $\mathrm{cKO}$ mice, immunofluorescence of plakoglobin was increased, $\alpha$ catenin decreased, and desmoplakin was found at EC contacts but not at those of control mice. The shift of molecular composition of junctional proteins in the $\beta$-catenin $\mathrm{cKO}$ mice has led to the authors' interesting hypothesis: lack of $\beta$-catenin forces creation of extra VE-cadherin/plakoglobin complexes and plakoglobin/desmoplakin/vimentin interaction analogous to that in complexus adhaerentes in lymphatic vessels [76]. In addition, proliferation of cultured ECs stimulated by VEGF receptor was not inhibited by confluency in the absence of $\beta$-catenin [75]. ECs express an array of Frizzled/Lrp (low-density lipoprotein receptorrelated protein) receptor complexes, a target of Wnt (Wingless and Int-1) ligand [77]. Canonical Wnt signaling includes $\beta$-catenin transcriptional activity through interaction with $\mathrm{T}$ cell factor (Tcf)/Lef transcription factors [78]. Wnt canonical system is involved in embryonic AG [79] and the vascular phenotype of $\beta$-catenin cKO mice may partially be a result of Wnt signal failure. Interestingly, the constitutively active $\beta$ catenin mutant in ECs also impairs embryonic AG because of overactivity of Notch-related pathways [80].
3.3. p120-Catenin. p120-Catenin stabilizes membrane localization of VE-cadherin by preventing endocytosis [81]. Deletion of p120-catenin in ECs of mice is also embryonically lethal starting at E12.5 (40\% of mutated embryos) [11]; however, some of them survive without obvious abnormalities. p120-Catenin deletion causes defects in microvasculature after E9.5; reduced microvascular density, disorganized vascular networks with impaired branching and blind-ending vessels, and hemorrhages were found in the brain and other organs. Pericytes recruitment, VE-cadherin, and N-cadherin expression were decreased. Cultured ECs from p120-catenin cKO mice exhibited proliferation deficiency.

3.4. Integrins. Integrins are large family of adhesive proteins that interact with extracellular matrix components and some adhesion molecules in the cell membranes and are critical for cell motility and survival [82]. They are heterodimers of $\alpha$ and $\beta$ subunits that upon activation cluster to form focal adhesions and assemble intracellular signaling molecules that are linked to actin cytoskeleton and specific cellular functions. In addition, integrins regulate VE-cadherin/catenin complexes during cell movement [83]. A vast number of reports show the importance of particular integrins in $\mathrm{AG}[8,84,85]$. Integrin $\alpha \mathrm{v} \beta 3$ is coreceptor of VEGF receptor in AG [86] and null or cKO mice (ECs) for several integrin subunits like $\alpha v, \alpha 3, \alpha 5, \beta 1$, and $\beta 3$ demonstrate their obligatory role in AG [87-89]. Deficiency of those subunits causes severe defects in vascular development: disturbed vessel organization, defective tubulogenesis, hemorrhage, vessel wall rupture, and embryonic death. Intracellular adaptor molecules linked to integrin dimers also control AG. Deletion of talin-1, which directly associates with $\beta$ subunit of integrins and mediates coupling to actin, in ECs of mice produced severe defect in AG with diffuse hemorrhages and disrupted vascular trees, leading to embryonic death [90]. Kindlin2 , the newly discovered $\beta$ subunit partner important for integrin activation [91], is also critical for AG. In mice, heterozygous deletion of kindlin-2 resulted in formation of immature vessels in implanted tumors. ECs from those mice showed reduction of integrin $\beta 3$-dependent adhesion, migration, and tube formation. Another key component of integrin activation, focal adhesion kinase (FAK), induced tumor AG [91].

Integrin $\alpha 9 \beta 1$ appears to have specific role in LAG. Integrin $\alpha 9$-null mice suffered insufficiency of the valves in collecting lymph vessels, developed chylothorax, and inexorably died by postnatal day 12 because of respiratory failure [92]. The valves had malformed leaflets due to missing integrin $\alpha 9 \beta 1 /$ fibronectin interaction that is necessary for the leaflets matrix organization [93]. Integrin $\alpha 9 \beta 1$ has unique property of binding directly VEGF to promote ECs adhesion and migration [94].

ECs in lymphatic microvasculature have specialized junctions that apparently reflect specific functionality of lymph capillaries [62]. Those "button-like" structures form discontinuous line of adhesions that allow easy flow of interstitial constituents into the lumen. During embryonic development ECs in lymphatic capillaries have continuous intercellular junctions that evolve to the "buttons" soon after birth [95]. 
The molecular composition of the junctions in lymphatic ECs is mixture of adherens and tight junctional proteins (VEcadherin, occluding, claudin, afadin, etc.). Contrary to the role of those proteins in AG, importance of actin-associated junctional molecules in LAG (except partially for afadin) remains to be elucidated.

\section{Necessity for Clarifying the Role of Similar Molecules in Developmental and Pathological AG and LAG}

Pathological AG and LAG are considered dysregulated processes utilizing similar molecular repertoire as AG and LAG during embryogenesis [4]. However, there is still little knowledge about the specificity of signaling mechanisms in pathological AG and LAG. Available reports have demonstrated altered functionality of certain angiogenic molecules like placental growth factor (PIGF), VEGF receptor-1, and VEGF-B in the context of diseased state [96-98]. Those pathology-associated investigations of the key angiogenic molecules such as VEGF ligands and receptors are the actual basis for understanding and eventually discovering the targets for antiangiogenic therapy. Actin-related junctional molecules are probably not exception and they may have additional/changed functionality in diseased conditions: for example, VE-cadherin in neoplastic vascular ECs expresses epitope that normally is obscured and this molecular segment allows specific targeting of tumor ECs [99]. Promising objective for antiangiogenic therapy especially in cancer is integrin $\alpha v \beta 3$, essential molecule for tumor AG. At present, several groups of pharmacological inhibitors of integrin $\alpha \mathrm{v} \beta 3$ are in the different stages of testing and the results show favorable effects in preclinical and clinical settings [100].

\section{Conclusion}

AG and LAG are attractive targets for influencing pathological conditions that present with excessive/abnormal vascular proliferation (chronic inflammation, cancer) or ischemia (atherosclerosis). The growth of vessels in AG demands fundamental processes of cell biology and morphogenesis: cellular adhesion, migration, proliferation, and survival. All of these functions utilize as essential effector actin filaments and associated junctional molecules that assure intercellular or cell-matrix communication. As a part of the same machinery, actin-tethered molecular complexes appear to be highly integrated, and they cooperate to regulate organization or disorganization of particular adhesion structure. Furthermore, the main switch of AG, VEGF signaling, is intimately dependent on these molecular complexes. Because of these properties, molecular assemblies including VE-cadherin, integrin, and nectin as well as actin may be considered as (co)targets of VEGF-related pro- or antiangiogenic therapy.

Afadin functions downstream of angiogenic signals translated by VEGF and S1P in ECs and also may represent target for modulating AG. Afadin has multiple roles in cellular processes, which may broaden the effects of its inhibition. Not only physiological AG but also pathological
AG (e.g., neovascularization in tumors) might be affected by modulating the afadin-related signals. However, there are many unanswered questions at present to justify this strategy: compromising intercellular adhesions may promote metastasis in cancer, whereas dysfunctional actin-related machinery might have opposite effects by preventing cell adhesion and promoting apoptosis; fundamental roles of cell junctions most probably will require targeted approach, delivery of active substances only to the diseased location; mechanisms involving afadin in pathological AG could have altered effects. Nevertheless, the proposed hypotheses might be worth testing in translational studies with hope that it will help in reducing the resistance to VEGF receptor inhibitors in VEGF-induced (or other) pathological AG.

\section{Conflict of Interests}

All the authors declare that there is no conflict of interests regarding the publication of this paper.

\section{References}

[1] W. C. Aird, "Phenotypic heterogeneity of the endothelium: I. Structure, function, and mechanisms," Circulation Research, vol. 100, no. 2, pp. 158-173, 2007.

[2] K. L. Marcelo, L. C. Goldie, and K. K. Hirschi, "Regulation of endothelial cell differentiation and specification," Circulation Research, vol. 112, no. 9, pp. 1272-1287, 2013.

[3] L. Coultas, K. Chawengsaksophak, and J. Rossant, "Endothelial cells and VEGF in vascular development," Nature, vol. 438, no. 7070, pp. 937-945, 2005.

[4] A. S. Chung and N. Ferrara, "Developmental and pathological angiogenesis," Annual Review of Cell and Developmental Biology, vol. 27, pp. 563-584, 2011.

[5] T. Tammela and K. Alitalo, "Lymphangiogenesis: molecular mechanisms and future promise," Cell, vol. 140, no. 4, pp. 460476, 2010.

[6] S. Schulte-Merker, A. Sabine, and T. V. Petrova, "Lymphatic vascular morphogenesis in development, physiology, and disease," The Journal of Cell Biology, vol. 193, no. 4, pp. 607-618, 2011.

[7] E. Dejana, "Endothelial cell-cell junctions: happy together," Nature Reviews Molecular Cell Biology, vol. 5, no. 4, pp. 261-270, 2004.

[8] C. Rüegg and A. Mariotti, "Vascular integrins: pleiotropic adhesion and signaling molecules in vascular homeostasis and angiogenesis," Cellular and Molecular Life Sciences, vol. 60, no. 6, pp. 1135-1157, 2003.

[9] G. H. Mahabeleshwar, W. Feng, K. Reddy, E. F. Plow, and T. V. Byzova, "Mechanisms of integrin-vascular endothelial growth factor receptor cross-activation in angiogenesis," Circulation Research, vol. 101, no. 6, pp. 570-580, 2007.

[10] A. Cattelino, S. Liebner, R. Gallini et al., "The conditional inactivation of the $\beta$-catenin gene in endothelial cells causes a defective vascular pattern and increased vascular fragility," The Journal of Cell Biology, vol. 162, no. 6, pp. 1111-1122, 2003.

[11] R. G. Oas, K. Xiao, S. Summers et al., "P120-catenin is required for mouse vascular development," Circulation Research, vol. 106, no. 5, pp. 941-951, 2010. 
[12] Y. Luo and G. L. Radice, "N-cadherin acts upstream of VEcadherin in controlling vascular morphogenesis," The Journal of Cell Biology, vol. 169, no. 1, pp. 29-34, 2005.

[13] K. J. Bayless and G. A. Johnson, "Role of the cytoskeleton in formation and maintenance of angiogenic sprouts," Journal of Vascular Research, vol. 48, no. 5, pp. 369-385, 2011.

[14] H. Schnittler, M. Taha, M. O. Schnittler, A. A. Taha, N. Lindemann, and J. Seebach, "Actin filament dynamics and endothelial cell junctions: the Ying and Yang between stabilization and motion," Cell and Tissue Research, vol. 355, no. 3, pp. 529-543, 2014.

[15] H. Ogita, Y. Rikitake, J. Miyoshi, and Y. Takai, "Cell adhesion molecules nectins and associating proteins: Implications for physiology and pathology," Proceedings of the Japan Academy Series B: Physical and Biological Sciences, vol. 86, no. 6, pp. 621629, 2010.

[16] C. Kim, H. Yang, Y. Fukushima et al., "Vascular RhoJ is an effective and selective target for tumor angiogenesis and vascular disruption," Cancer Cell, vol. 25, no. 1, pp. 102-117, 2014.

[17] D. Gerald, I. Adini, S. Shechter et al., "RhoB controls coordination of adult angiogenesis and lymphangiogenesis following injury by regulating VEZF1-mediated transcription," Nature Communications, vol. 4, article 2824, 2013.

[18] B. A. Bryan, E. Dennstedt, D. C. Mitchell et al., "RhoA/ROCK signaling is essential for multiple aspects of VEGF-mediated angiogenesis," The FASEB Journal, vol. 24, no. 9, pp. 3186-3195, 2010.

[19] N. Ferrara, H.-P. Gerber, and J. LeCouter, "The biology of VEGF and its receptors," Nature Medicine, vol. 9, no. 6, pp. 669-676, 2003.

[20] K. Mandai, H. Nakanishi, A. Satoh et al., "Afadin: a novel actin filament-binding protein with one PDZ domain localized at cadherin-based cell-to-cell adherens junction," The Journal of Cell Biology, vol. 139, no. 2, pp. 517-528, 1997.

[21] Y. Takai and H. Nakanishi, "Nectin and afadin: novel organizers of intracellular junctions," Journal of Cell Science, vol. 116, no. 1, pp. 17-27, 2003.

[22] Y. Takai, K. Irie, K. Shimizu, T. Sakisaka, and W. Ikeda, "Nectins and nectin-like molecules: roles in cell adhesion, migration, and polarization," Cancer Science, vol. 94, no. 8, pp. 655-667, 2003.

[23] M. Miyata, H. Ogita, H. Komura et al., "Localization of nectinfree afadin at the leading edge and its involvement in directional cell movement induced by platelet-derived growth factor," Journal of Cell Science, vol. 122, no. 23, pp. 4319-4329, 2009.

[24] Y. Fukumoto, S. Kurita, Y. Takai, and H. Ogita, "Role of scaffold protein afadin dilute domain-interacting protein (ADIP) in platelet-derived growth factor-induced cell movement by activating Rac protein through Vav2 protein," The Journal of Biological Chemistry, vol. 286, no. 50, pp. 43537-43548, 2011.

[25] T. Majima, H. Ogita, T. Yamada et al., "Involvement of afadin in the formation and remodeling of synapses in the hippocampus," Biochemical and Biophysical Research Communications, vol. 385, no. 4, pp. 539-544, 2009.

[26] J.-E. van Leeuwen, I. Rafalovich, K. Sellers et al., "Coordinated nuclear and synaptic shuttling of afadin promotes spine plasticity and histone modifications," The Journal of Biological Chemistry, vol. 289, no. 15, pp. 10831-10842, 2014.

[27] B. M. Gumbiner, "Cell adhesion: the molecular basis of tissue architecture and morphogenesis," Cell, vol. 84, no. 3, pp. 345357, 1996.
[28] A. B. Zhadanov, D. W. Provance Jr., C. A. Speer et al., "Absence of the tight junctional protein AF-6 disrupts epithelial cell-cell junctions and cell polarity during mouse development," Current Biology, vol. 9, no. 16, pp. 880-888, 1999.

[29] G. Fournier, O. Cabaud, E. Josselin et al., "Loss of AF6/afadin, a marker of poor outcome in breast cancer, induces cell migration, invasiveness and tumor growth," Oncogene, vol. 30, no. 36, pp. 3862-3874, 2011.

[30] G. M. J. Beaudoin III, C. M. Schofield, T. Nuwal et al., "Afadin, a Ras/Rap effector that controls cadherin function, promotes spine and excitatory synapse density in the hippocampus," The Journal of Neuroscience, vol. 32, no. 1, pp. 99-110, 2012.

[31] M. Tanaka-Okamoto, K. Hori, H. Ishizaki et al., "Involvement of afadin in barrier function and homeostasis of mouse intestinal epithelia," Journal of Cell Science, vol. 124, no. 13, pp. 2231-2240, 2011.

[32] S. Elloul, D. Kedrin, N. W. Knoblauch, A. H. Beck, and A. Toker, "The adherens junction protein Afadin is an AKT substrate that regulates breast cancer cell migration," Molecular Cancer Research, vol. 12, no. 3, pp. 464-476, 2014.

[33] M. Potente, H. Gerhardt, and P. Carmeliet, "Basic and therapeutic aspects of angiogenesis," Cell, vol. 146, no. 6, pp. 873-887, 2011.

[34] P. Carmeliet, "Mechanisms of angiogenesis and arteriogenesis," Nature Medicine, vol. 6, no. 4, pp. 389-395, 2000.

[35] R. H. Adams and K. Alitalo, "Molecular regulation of angiogenesis and lymphangiogenesis," Nature Reviews Molecular Cell Biology, vol. 8, no. 6, pp. 464-478, 2007.

[36] M. Jeltsch, V.-M. Leppänen, P. Saharinen, and K. Alitalo, "Receptor tyrosine kinase-mediated angiogenesis," Cold Spring Harbor Perspectives in Biology, vol. 5, no. 9, Article ID a009183, 2013.

[37] P. Carmeliet, V. Ferreira, G. Breier et al., "Abnormal blood vessel development and lethality in embryos lacking a single VEGF allele," Nature, vol. 380, no. 6573, pp. 435-439, 1996.

[38] G.-H. Fong, J. Rossant, M. Gertsenstein, and M. L. Breitman, "Role of the Flt-1 receptor tyrosine kinase in regulating the assembly of vascular endothelium," Nature, vol. 376, no. 6535, pp. 66-70, 1995.

[39] F. Shalaby, J. Rossant, T. P. Yamaguchi et al., "Failure of bloodisland formation and vasculogenesis in Flk-1-deficient mice," Nature, vol. 376, no. 6535, pp. 62-66, 1995.

[40] S. Moens, J. Goveia, P. C. Stapor, A. R. Cantelmo, and P. Carmeliet, "The multifaceted activity of VEGF in angiogenesis-implications for therapy responses," Cytokine \& Growth Factor Reviews, vol. 25, no. 4, pp. 473-482, 2014.

[41] M. R. H. Kooistra, N. Dubé, and J. L. Bos, "Rapl: a key regulator in cell-cell junction formation," Journal of Cell Science, vol. 120, no. 1, pp. 17-22, 2007.

[42] T. Sato, N. Fujita, A. Yamada et al., "Regulation of the assembly and adhesion activity of E-cadherin by nectin and afadin for the formation of adherens junctions in Madin-Darby canine kidney cells," The Journal of Biological Chemistry, vol. 281, no. 8, pp. 5288-5299, 2006.

[43] H. Tawa, Y. Rikitake, M. Takahashi et al., "Role of afadin in vascular endothelial growth factor-and sphingosine 1-phosphateinduced angiogenesis," Circulation Research, vol. 106, no. 11, pp. 1731-1742, 2010.

[44] Y. Rikitake, K.-I. Hirata, S. Kawashima et al., "Involvement of endothelial nitric oxide in sphingosine-1-phosphate-induced angiogenesis," Arteriosclerosis, Thrombosis, and Vascular Biology, vol. 22, no. 1, pp. 108-114, 2002. 
[45] E. Ackah, J. Yu, S. Zoellner et al., "Akt1/protein kinase B $\alpha$ is critical for ischemic and VEGF-mediated angiogenesis," The Journal of Clinical Investigation, vol. 115, no. 8, pp. 2119-2127, 2005.

[46] A. C. Monteiro, R. Sumagin, C. R. Rankin et al., "JAM-A associates with ZO-2, afadin, and PDZ-GEF1 to activate Rap2c and regulate epithelial barrier function," Molecular Biology of the Cell, vol. 24, no. 18, pp. 2849-2860, 2013.

[47] A. A. Birukova, X. Tian, Y. Tian, K. Higginbotham, and K. G. Birukov, "Rap-afadin axis in control of Rho signaling and endothelial barrier recovery," Molecular Biology of the Cell, vol. 24, no. 17, pp. 2678-2688, 2013.

[48] A. A. Birukova, P. Fu, T. Wu et al., "Afadin controls p120-catenin-ZO-1 interactions leading to endothelial barrier enhancement by oxidized phospholipids," Journal of Cellular Physiology, vol. 227, no. 5, pp. 1883-1890, 2012.

[49] Z. Yang, S. Zimmerman, P. R. Brakeman, G. M. Beaudoin, L. F. Reichardt, and D. K. Marciano, "De novo lumen formation and elongation in the developing nephron: a central role for afadin in apical polarity," Development, vol. 140, no. 8, pp. 1774-1784, 2013.

[50] E. A. Severson, W. Y. Lee, C. T. Capaldo, A. Nusrat, and C. A. Parkos, "Junctional adhesion molecule a interacts with afadin and PDZ-GEF2 to activate RaplA, regulate $\beta 1$ integrin levels, and enhance cell migration," Molecular Biology of the Cell, vol. 20, no. 7, pp. 1916-1925, 2009.

[51] M. Chrzanowska-Wodnicka, A. E. Kraus, D. Gale, G. C. White II, and J. Vansluys, "Defective angiogenesis, endothelial migration, proliferation, and MAPK signaling in Raplb-deficient mice," Blood, vol. 111, no. 5, pp. 2647-2656, 2008.

[52] J. S. Mudgett, J. Ding, L. Guh-Siesel et al., "Essential role for p38 $\alpha$ mitogen-activated protein kinase in placental angiogenesis," Proceedings of the National Academy of Sciences of the United States of America, vol. 97, no. 19, pp. 10454-10459, 2000.

[53] N. Hatano, Y. Mori, M. Oh-hora et al., "Essential role for ERK2 mitogen-activated protein kinase in placental development," Genes to Cells, vol. 8, no. 11, pp. 847-856, 2003.

[54] M. Chrzanowska-Wodnicka, S. S. Smyth, S. M. Schoenwaelder, T. H. Fischer, and G. C. White II, "Raplb is required for normal platelet function and hemostasis in mice," The Journal of Clinical Investigation, vol. 115, no. 3, pp. 680-687, 2005.

[55] K. Katagiri, A. Maeda, M. Shimonaka, and T. Kinashi, "RAPL, a Rapl-binding molecule that mediates Rapl-induced adhesion through spatial regulation of LFA-1," Nature Immunology, vol. 4, no. 8, pp. 741-748, 2003.

[56] G. Carmona, S. Göttig, A. Orlandi et al., "Role of the small GTPase Rapl for integrin activity regulation in endothelial cells and angiogenesis," Blood, vol. 113, no. 2, pp. 488-497, 2009.

[57] T. Majima, K. Takeuchi, K. Sano et al., "An adaptor molecule afadin regulates lymphangiogenesis by modulating RhoA activity in the developing mouse embryo," PLoS ONE, vol. 8, no. 6, Article ID e68134, 2013.

[58] J. Waschke, F. E. Curry, R. H. Adamson, and D. Drenckhahn, "Regulation of actin dynamics is critical for endothelial barrier functions," American Journal of Physiology-Heart and Circulatory Physiology, vol. 288, no. 3, pp. H1296-H1305, 2005.

[59] K. J. Whitehead, A. C. Chan, S. Navankasattusas et al., "The cerebral cavernous malformation signaling pathway promotes vascular integrity via Rho GTPases," Nature Medicine, vol. 15, no. 2, pp. 177-184, 2009.
[60] M. R. H. Kooistra, M. Corada, E. Dejana, and J. L. Bos, "Epacl regulates integrity of endothelial cell junctions through VEcadherin," FEBS Letters, vol. 579, no. 22, pp. 4966-4972, 2005.

[61] K. Noda, J. Zhang, S. Fukuhara, S. Kunimoto, M. Yoshimura, and N. Mochizuki, "Vascular endothelial-cadherin stabilizes at cell-cell junctions by anchoring to circumferential actin bundles through $\alpha$ - and $\beta$-catenins in cyclic AMP-Epac-Rap1 signalactivated endothelial cells," Molecular Biology of the Cell, vol. 21, no. 4, pp. 584-596, 2010.

[62] P. Baluk, J. Fuxe, H. Hashizume et al., "Functionally specialized junctions between endothelial cells of lymphatic vessels," The Journal of Experimental Medicine, vol. 204, no. 10, pp. 23492362, 2007.

[63] N. Kanzaki, H. Ogita, H. Komura et al., "Involvement of the nectin-afadin complex in PDGF-induced cell survival," Journal of Cell Science, vol. 121, no. 12, pp. 2008-2017, 2008.

[64] N. Rudini and E. Dejana, "Adherens junctions," Current Biology, vol. 18, no. 23, pp. R1080-R1082, 2008.

[65] J. E. Nieset, A. R. Redfield, F. Jin, K. A. Knudsen, K. R. Johnson, and M. J. Wheelock, "Characterization of the interactions of $\alpha$ catenin with $\alpha$-actinin and $\beta$-catenin/plakoglobin," Journal of Cell Science, vol. 110, no. 8, pp. 1013-1022, 1997.

[66] L. Shapiro and W. I. Weis, "Structure and biochemistry of cadherins and catenins," Cold Spring Harbor Perspectives in Biology, vol. 1, no. 3, Article ID a003053, 2009.

[67] A. B. Reynolds and R. H. Carnahan, "Regulation of cadherin stability and turnover by p120ctn: implications in disease and cancer," Seminars in Cell and Developmental Biology, vol. 15, no. 6, pp. 657-663, 2004.

[68] P. Carmeliet, M.-G. Lampugnani, L. Moons et al., "Targeted deficiency or cytosolic truncation of the VE-cadherin gene in mice impairs VEGF-mediated endothelial survival and angiogenesis," Cell, vol. 98, no. 2, pp. 147-157, 1999.

[69] I. Shiojima and K. Walsh, "Role of Akt signaling in vascular homeostasis and angiogenesis," Circulation Research, vol. 90, no. 12, pp. 1243-1250, 2002.

[70] M. Corada, M. Mariotti, G. Thurston et al., "Vascular endothelial-cadherin is an important determinant of microvascular integrity in vivo," Proceedings of the National Academy of Sciences of the United States of America, vol. 96, no. 17, pp. 98159820, 1999.

[71] A. C. Rapraeger, B. J. Ell, M. Roy et al., "Vascular endothelialcadherin stimulates syndecan-1-coupled insulin-like growth factor-1 receptor and cross-talk between $\alpha v \beta 3$ integrin and vascular endothelial growth factor receptor 2 at the onset of endothelial cell dissemination during angiogenesis," FEBS Journal, vol. 280, no. 10, pp. 2194-2206, 2013.

[72] M. Hellström, L.-K. Phng, J. J. Hofmann et al., "Dll4 signalling through Notchl regulates formation of tip cells during angiogenesis," Nature, vol. 445, no. 7129, pp. 776-780, 2007.

[73] L. Jakobsson, C. A. Franco, K. Bentley et al., "Endothelial cells dynamically compete for the tip cell position during angiogenic sprouting," Nature Cell Biology, vol. 12, no. 10, pp. 943-953, 2010.

[74] K. Bentley, C. A. Franco, A. Philippides et al., "The role of differential VE-cadherin dynamics in cell rearrangement during angiogenesis," Nature Cell Biology, vol. 16, no. 4, pp. 309321, 2014.

[75] M. G. Lampugnani, A. Zanetti, M. Corada et al., "Contact inhibition of VEGF-induced proliferation requires vascular endothelial cadherin, $\beta$-catenin, and the phosphatase DEP-1/ CD148," The Journal of Cell Biology, vol. 161, no. 4, pp. 793-804, 2003. 
[76] B. Hämmerling, C. Grund, J. Boda-Heggemann, R. Moll, and W. W. Franke, "The Complexus adhaerens of mammalian lymphatic endothelia revisited: a junction even more complex than hitherto thought," Cell and Tissue Research, vol. 324, no. 1, pp. 55-67, 2006.

[77] A. M. Goodwin, K. M. Sullivan, and P. A. D’Amore, “Cultured endothelial cells display endogenous activation of the canonical Wnt signaling pathway and express multiple ligands, receptors, and secreted modulators of Wnt signaling," Developmental Dynamics, vol. 235, no. 11, pp. 3110-3120, 2006.

[78] H. Clevers, "Wnt/ $\beta$-Catenin Signaling in Development and Disease," Cell, vol. 127, no. 3, pp. 469-480, 2006.

[79] C. A. Franco, S. Liebner, and H. Gerhardt, "Vascular morphogenesis: a Wnt for every vessel?” Current Opinion in Genetics and Development, vol. 19, no. 5, pp. 476-483, 2009.

[80] M. Corada, D. Nyqvist, F. Orsenigo et al., "The Wnt/ $\beta$-catenin pathway modulates vascular remodeling and specification by upregulating Dll4/notch signaling," Developmental Cell, vol. 18, no. 6, pp. 938-949, 2010.

[81] C. M. Chiasson, K. B. Wittich, P. A. Vincent, V. Faundez, and A. P. Kowalczyk, "P120-catenin inhibits VE-cadherin internalization through a Rho-independent mechanism," Molecular Biology of the Cell, vol. 20, no. 7, pp. 1970-1980, 2009.

[82] F. G. Giancotti and E. Ruoslahti, "Integrin signaling," Science, vol. 285, no. 5430, pp. 1028-1032, 1999.

[83] Y. Wang, G. Jin, H. Miao, J. Y. S. Li, S. Usami, and S. Chien, "Integrins regulate VE-cadherin and catenins: dependence of this regulation on Src, but not on Ras," Proceedings of the National Academy of Sciences of the United States of America, vol. 103, no. 6, pp. 1774-1779, 2006.

[84] N. L. Malinin, E. Pluskota, and T. V. Byzova, "Integrin signaling in vascular function," Current Opinion in Hematology, vol. 19, no. 3, pp. 206-211, 2012.

[85] C. J. Avraamides, B. Garmy-Susini, and J. A. Varner, "Integrins in angiogenesis and lymphangiogenesis," Nature Reviews Cancer, vol. 8, no. 8, pp. 604-617, 2008.

[86] P. R. Somanath, N. L. Malinin, and T. V. Byzova, "Cooperation between integrin $\alpha v \beta 3$ and VEGFR2 in angiogenesis," Angiogenesis, vol. 12, no. 2, pp. 177-185, 2009.

[87] A. C. Zovein, A. Luque, K. A. Turlo et al., “ $\beta 1$ integrin establishes endothelial cell polarity and arteriolar lumen formation via a Par3-dependent mechanism," Developmental Cell, vol. 18, no. 1, pp. 39-51, 2010.

[88] A. van der Flier, K. Badu-Nkansah, C. A. Whittaker et al., "Endothelial $\alpha 5$ and $\alpha \mathrm{v}$ integrins cooperate in remodeling of the vasculature during development," Development, vol. 137, no. 14, pp. 2439-2449, 2010.

[89] K. M. Hodivala-Dilke, K. P. McHugh, D. A. Tsakiris et al., “ $\beta 3$ integrin-deficient mice are a model for Glanzmann thrombasthenia showing placental defects and reduced survival," Journal of Clinical Investigation, vol. 103, no. 2, pp. 229-238, 1999.

[90] S. J. Monkley, V. Kostourou, L. Spence et al., "Endothelial cell talin1 is essential for embryonic angiogenesis," Developmental Biology, vol. 349, no. 2, pp. 494-502, 2011.

[91] M. Moser, K. R. Legate, R. Zent, and R. Fässler, "The tail of integrins, talin, and kindlins," Science, vol. 324, no. 5929, pp. 895-899, 2009.

[92] X. Z. Huang, J. F. Wu, R. Ferrando et al., "Fatal bilateral chylothorax in mice lacking the integrin $\alpha 9 \beta 1$," Molecular and Cellular Biology, vol. 20, no. 14, pp. 5208-5215, 2000.
[93] E. Bazigou, S. Xie, C. Chen et al., "Integrin- $\alpha 9$ is required for fibronectin matrix assembly during lymphatic valve morphogenesis," Developmental Cell, vol. 17, no. 2, pp. 175-186, 2009.

[94] N. E. Vlahakis, B. A. Young, A. Atakilit et al., "Integrin $\alpha 9 \beta 1$ directly binds to vascular endothelial growth factor (VEGF)-A and contributes to VEGF-A-induced angiogenesis," The Journal of Biological Chemistry, vol. 282, no. 20, pp. 15187-15196, 2007.

[95] L.-C. Yao, P. Baluk, R. S. Srinivasan, G. Oliver, and D. M. McDonald, "Plasticity of button-like junctions in the endothelium of airway lymphatics in development and inflammation," The American Journal of Pathology, vol. 180, no. 6, pp. 2561-2575, 2012.

[96] M. Dewerchin and P. Carmeliet, "PlGF: a multitasking cytokine with disease-restricted activity," Cold Spring Harbor Perspectives in Medicine, vol. 2, no. 8, Article ID a011056, 2012.

[97] E. Boscolo, J. B. Mulliken, and J. Bischoff, "VEGFR-1 mediates endothelial differentiation and formation of blood vessels in a murine model of infantile hemangioma," The American Journal of Pathology, vol. 179, no. 5, pp. 2266-2277, 2011.

[98] R. Serpi, A. M. Tolonen, J. Huusko et al., "Vascular endothelial growth factor-B gene transfer prevents angiotensin II-induced diastolic dysfunction via proliferation and capillary dilatation in rats," Cardiovascular Research, vol. 89, no. 1, pp. 204-213, 2011.

[99] C. May, J. R. Doody, R. Abdullah et al., "Identification of a transiently exposed VE-cadherin epitope that allows for specific targeting of an antibody to the tumor neovasculature," Blood, vol. 105, no. 11, pp. 4337-4344, 2005.

[100] Z. Liu, F. Wang, and X. Chen, "Integrin $\alpha \mathrm{v} \beta 3$-targeted cancer therapy," Drug Development Research, vol. 69, no. 6, pp. 329-339, 2008. 


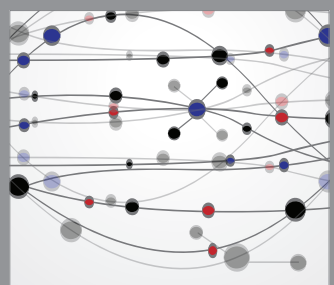

The Scientific World Journal
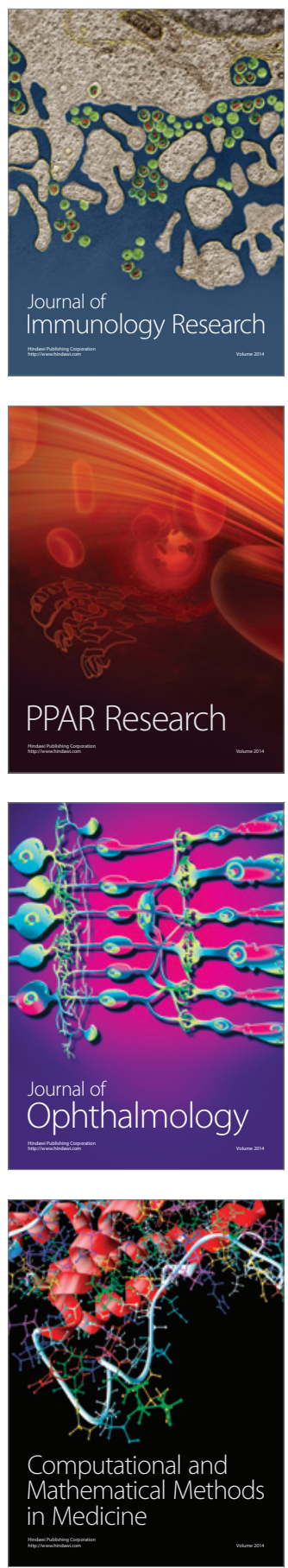

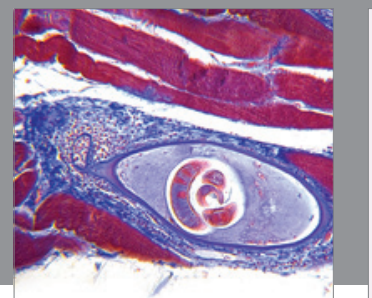

Gastroenterology

Research and Practice
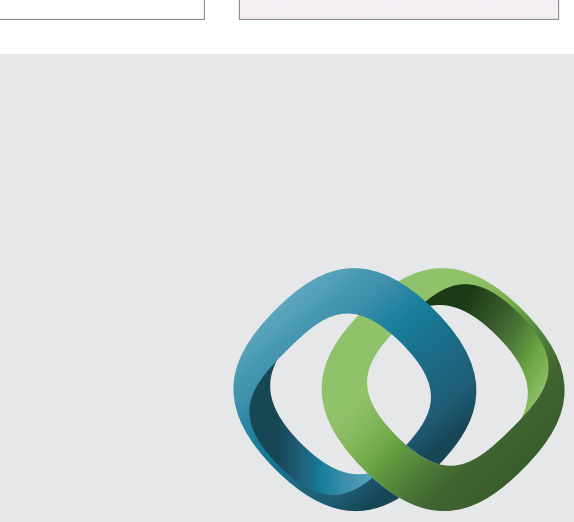

\section{Hindawi}

Submit your manuscripts at

http://www.hindawi.com
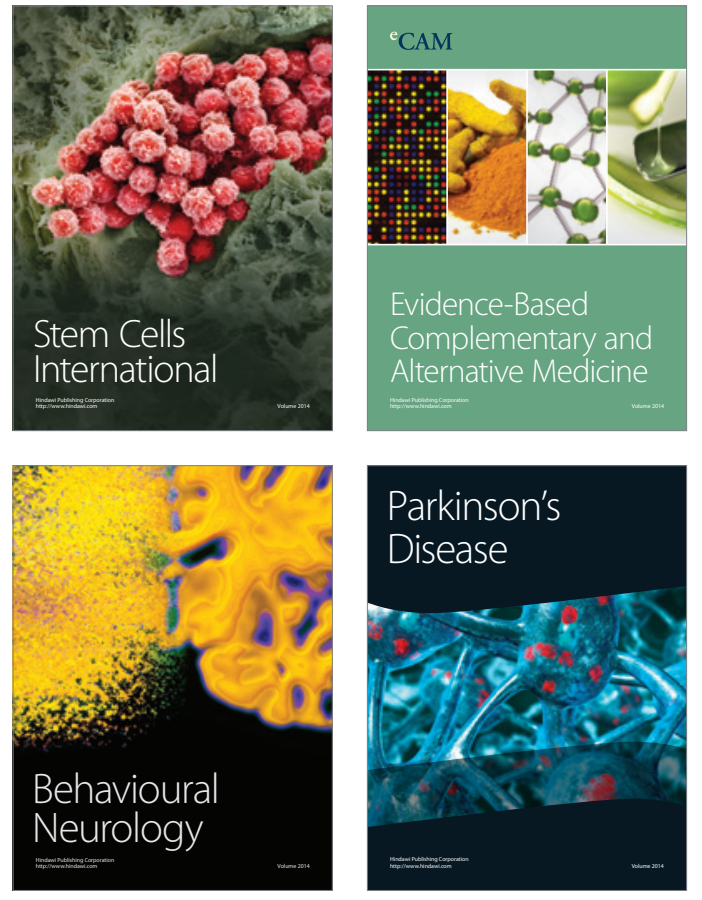
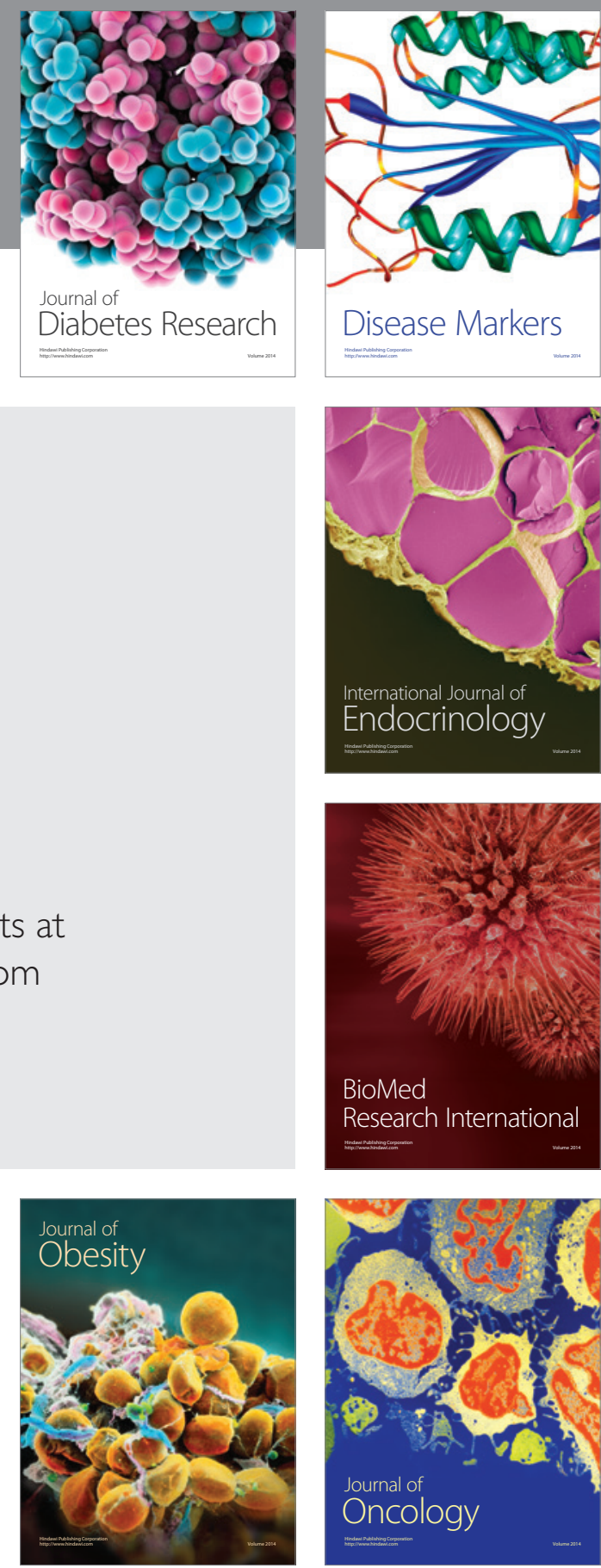

Disease Markers
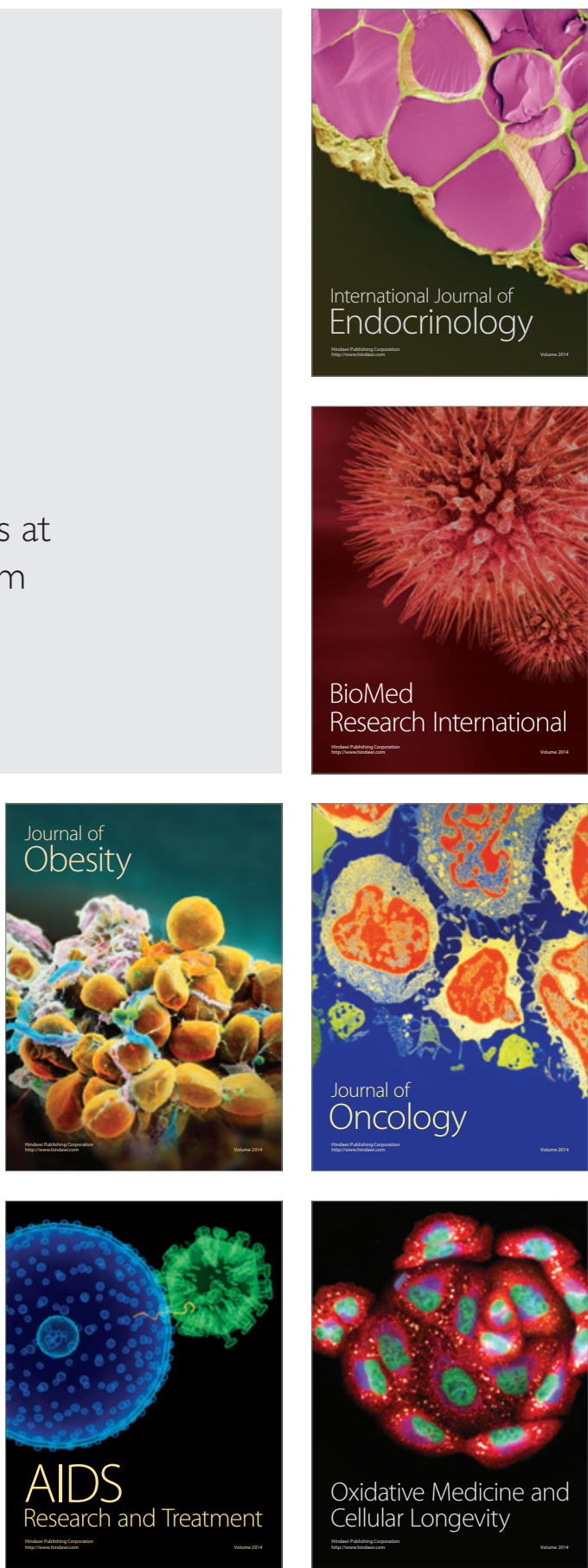